\title{
Furan-2,5-dicarboxylic acid, a promising platform molecule: polymer, monomer, and MOF
}

\author{
Yimin Mao ${ }^{a}$, Peter Zavalij ${ }^{b}$ \\ a Department of Materials Science and Engineering, University of Maryland, College \\ Park, Maryland 20742, USA; and NIST Center for Neutron Research, National \\ Institute of Standards and Technology, Gaithersburg, Maryland 20899, USA, \\ yimin.mao@nist.gov \\ b Department of Chemistry and Biochemistry, University of Maryland, College Park, \\ Maryland 20742, USA.
}

Furan-2,5-dicarboxylic acid (FDCA), a small molecule discovered more than 100 years ago has been recently attracting attentions from both academic and industrial communities. Large-scale, economic production of FDCA has been accomplished, utilizing biomass fermentation residues, thanks to the breakthroughs in synthesis. [1] It has been ranked as one of the top platform molecules that are promising to produce functional materials via green chemistry. We report recent structural studies on three aspects of FDCA-based materials and their potential applications.

In the first case, crystal structure of a polymer, poly(ethylene furanoate) (PEF) which is a type of linear homopolymer of FDCA, is determined in a semi-quantitative way, using X-ray fiber diffraction method. Via 2D analysis of fiber diffraction pattern a monoclinic unit cell containing two staggered chains is proposed. [2] The chain staggering is attributed to the superior gas barrier properties of the solid-state PEF film. Secondly, co-crystallization of FDCA with polar aprotic solvents, namely, dimethyl sulfoxide (DMSO) and dimethylformamide (DMF) is investigated by using single-crystal crystallography. The two types co-crystals exhibit distinct hydrogenbonding networks. Moreover, crystal structures of the FDCA-DMSO solvate provides a fingerprint of sulfur-oxygen interaction. [3] This weak specific interaction is further investigated by using X-ray pair distribution function (PDF) method and FTIR spectroscopy. Last but not least, 1D, 2D, and 3D metal organic frameworks synthesized by employing FDCA as linker and $\mathrm{Zn}$ (II) as central ion is reported. [4] We demonstrate that FDCA can be used as a versatile linker to produce coordinate polymers.

\section{Reference}

[1] Jong, E. de, Dam et al. (2012). Biobased Monomers, Polymers, and Materials, edited by P. B. Smith \& R. A. Gross, pp. 1-13. ACS Symposium Series. Washington, DC: American Chemical Society.

[2] Mao, Y. et al. (2016). Polymer, 102, 308-314.

[3] Mao, Y. \& Zavalij, P. Y. (2018). Acta Cryst. C74, 986-990.

[4] Mao, Y. \& Zavalij, P. Y. (2019). Acta Cryst. C74, 1719-1724. 